\title{
Nonlinear Waves in Reaction Diffusion Systems: The Effect of Transport Memory
}

\author{
K. K. Manne' ${ }^{1}$ A. J. Hurd ${ }^{2}$, and V. M. Kenkre ${ }^{1}$ \\ 1 Center for Advanced Studies and Department of Physics and Astronomy, \\ University of New Mexico, Albuquerque, NM 87131 \\ 2 Sandia National Laboratories, \\ Albuquerque, NM 87185 \\ (October 15, 1999)
}

\begin{abstract}
Motivated by the problem of determining stress distributions in granular materials, we study the effect of finite transport correlation times on the propagation of nonlinear wavefronts in reaction diffusion systems. We obtain new results such as the possibility of spatial oscillations in the wavefront shape for certain values of the system parameters and high enough wavefront speeds. We also generalize earlier known results concerning the minimum wavefront speed and shape-speed relationships stemming from the finiteness of the correlation times. Analytic investigations are made possible by a piece-wise linear representation of the nonlinearity.
\end{abstract}

\section{INTRODUCTION}

RECEIVED NOV 291999

Well-known in the nonlinear literature is the logistic equation which, in its continuum form

$$
\frac{d u}{d t}=k u(b-u)
$$

describes the time evolution of the density of quasiparticles or the population of a species $u(t)$, which increases (linearly) due to some mechanism and decreases (quadratically) due to another, $k$ and $b$ being appropriate constants. Ubiquitous in traditional transport is the diffusion equation

$$
\frac{\partial u}{\partial t}=D \frac{\partial^{2} u}{\partial x^{2}}
$$

which describes diffusive transport of the quantity $u(x, t)$ in space, $D$ being the diffusion constant. Solutions of (I.1) or (I.2) are well known analytically for arbitrary initial conditions, but solutions of the Fisher equation $[1,2]$

$$
\frac{\partial u}{\partial t}=D \frac{\partial^{2} u}{\partial x^{2}}+k u(b-u)
$$

which combines (I.1) and (I.2), are not. The study of (I.3), and of its generalization

$$
\frac{\partial u}{\partial t}=D \frac{\partial^{2} u}{\partial x^{2}}+k f(u)
$$

where $f(u)$ is a nonlinear function, belongs to the area of reaction diffusion. There has been a lot of work in the area, much of it numerical. For instance, it is known that nonlinear wave fronts form which maintain their shape despite (in some sense because of the diffusive element of the evolution, contrary to linear intuition. It is also known that stable traveling wave solutions exist with speeds equaling or exceeding a minimum value; that if $f(u)$ has two zeros, at $u=0$ and $u=b$, as in the case being investigated in the present paper, an initial shape which on the two sides of a finite segment in space equals the two zeros of $f(u)$ respectively, evolves in time to the traveling wavefront form; that the speed is related to the steepness of the wavefront, slower speeds corresponding to steeper slopes; and that the minimum speed is given by $2 \sqrt{k D f^{\prime}(b)}$.

Our interest in the present paper is in a generalization of (1.4) to include transport memory effects, i.e., effects which arise if the linear part of the evolution represents a process which is in part ballistic and in part diffusive. The memory function or correlation function which describes the transport is, in such cases, not a $\delta$-function as in the purely diffusive case of (1.4), but has a finite decay time [3]. The diffusive process may arise from the motion and scattering of quasiparticles, the decay time being descriptive of the time between scattering events. The diffusion 


\section{DISCLAIMER}

This report was prepared as an account of work sponsored by an agency of the United States Government. Neither the United States Government nor any agency thereof, nor any of their employees, make any warranty, express or implied, or assumes any legal liability or responsibility for the accuracy, completeness, or usefulness of any information, apparatus, product, or process disclosed, or represents that its use would not infringe privately owned rights. Reference herein to any specific commercial product, process, or service by trade name, trademark, manufacturer, or otherwise does not necessarily constitute or imply its endorsement, recommendation, or favoring by the United States Government or any agency thereof. The views and opinions of authors expressed herein do not necessarily state or reflect those of the United States Government or any agency thereof. 


\section{DISCLAIMER}

Portions of this document may be illegible in electronic image products. Images are produced from the best available original document. 
equation describes the extreme situation in which that time is infinitesimally short. Since all realistic systems possess a finite scattering time, it is of interest to examine the intermediate, or general, case. Such general investigations of memory effects have resulted in advances in widely different areas such as exciton transport [3] and stress distribution [4].

The motivation for our investigation has come from the appearance of stress distribution equations with memory which arise in granular materials and will be commented upon in the conclusion section. Our studies lead to a generalization of results known in the extreme diffusive known earlier, as well as to additional and interesting new effects particular to finite correlation time systems.

\section{ANALYTIC APPROACH AND GENERAL SOLUTIONS}

Consider the replacement of the diffusion equation (I.2) by its nonlocal (in time) counterpart

$$
\frac{\partial u}{\partial t}=D \int_{0}^{t} \phi(t-s) \frac{\partial^{2} u(x, s)}{\partial s^{2}} d s
$$

where the so-called memory function $\phi(t)$ describes the finiteness of the correlation or scattering time [3]. For simplicity, let us take the memory to consist of a single exponential: $\phi(t)=\alpha e^{-\alpha t}$. The quantity $\alpha$ measures the reciprocal of the scattering or correlation time. In the limit $\alpha \rightarrow \infty$, the memory function becomes a $\delta$-function and one recovers the local diffusion equation, whereas, in the limit $\alpha \rightarrow 0, D \rightarrow \infty, \alpha D \rightarrow v^{2}$ (where $v$ is the wave speed), the memory function becomes a constant, and one obtains pure undamped linear waves. Finite $\alpha$ and $v$ describe the intermediate situation. The diffusion equation (I.2) is then replaced by the telegrapher's equation. Our starting point in this paper is the generalization of the usual reaction diffusion equation (I.4) to

$$
\frac{\partial^{2} u}{\partial t^{2}}+\alpha \frac{\partial u}{\partial t}=v^{2} \frac{\partial^{2} u}{\partial x^{2}}+s^{2} f(u)
$$

whose transport component is the telegrapher's equation rather than the diffusion equation. Here, $s$ is a constant which describes the strength of the nonlinearity in the system, and is related to $k$ in (I.4) through the limit $\alpha \rightarrow 0$, $s \rightarrow \infty, s^{2} / \alpha \rightarrow k$. The telegrapher's equation without the nonlinearity has been recently applied successfully [4] to the description of observations [6,7] of stress distributions in granular materials. In that application the vertical depth in the granular compact plays the role of time.

We are thus interested in investigating effects of finite correlation time (intermediate transport memory) on the propagation of nonlinear wave fronts. Our approach will be analytical rather than numerical. This is made possible by the assumption that the nonlinear function $f(u)$ is a piece-wise linear function. The logistic term in (I.3) may then be approximated by (see Fig. 1)

$$
f(u)= \begin{cases}\frac{u}{a} & u \leq a \\ \frac{b-u}{b-a} & u \geq a\end{cases}
$$

$a$ and $b$ being the values of $u$ at which the nonlinearity peaks and vanishes, respectively. Such a piece-wise linear approach has been introduced earlier [10] in the context of diffusive processes and has been shown to lead to analytical results for patterns in reaction diffusion systems. Our contribution in the present analysis is to apply a similar approach to investigate memory effects.

We do not address the general initial value problem related to (II.2). Instead, our interest is in finding only traveling wave solutions of (II.2). We will take them to move in the direction of increasing $x$ :

$$
u(x, t)=U(x-c t)=U(z)
$$

where $c$ is the speed of the nonlinear traveling wave (to be differentiated from the natural linear speed $v$ dictated by the medium). Denoting differentiation w.r.t. $z$ by primes, we reduce the partial differential equation (II.2) to an ordinary differential equation formally descriptive of a damped harmonic oscillator

$$
\begin{array}{ll}
m U^{\prime \prime}+2 \Gamma U^{\prime}+k_{1}^{2} U=0 & U \leq a \\
m U^{\prime \prime}+2 \Gamma U^{\prime}+k_{2}^{2}(b-U)=0 & U \geq a
\end{array}
$$


In equation (II.5), the mass $m$ of the fictitious oscillator arises from the difference in the wavefront speed and the linear wave speed dictated by the medium, while the frequency of the oscillator arises from the strength and shape of the nonlinearity. The parameters in (II.5) are given by

$$
m \equiv v^{2}-c^{2} ; \Gamma \equiv c \alpha / 2 ; k_{1}^{2} \equiv s^{2} / a ; k_{2}^{2} \equiv s^{2} /(b-a)
$$

The solution for the wave amplitude $U$ as a function of the coordinate $x-c t$ is available immediately in each of the two regions $U \leq a$ and $U \geq a$ [8]. In the region $U \leq a$, the solutions are

$$
U(z)=A e^{\frac{k_{1}^{2}}{2 \Gamma} z}
$$

if $m=0$ and

$$
U(z)= \begin{cases}A_{+} e^{r_{+} z}+A_{-} e^{r_{-} z} & r_{+} \neq r_{-} \\ A_{0} e^{r z}+A_{1} z e^{r z} & r_{+}=r_{-}=r\end{cases}
$$

if $m \neq 0$. Here,

$$
r_{ \pm}=\frac{-\Gamma \pm \sqrt{\Gamma^{2}-m k_{1}^{2}}}{m}
$$

In the region $U \geq a$ on the other hand,

$$
U(z)=b-C e^{\frac{k_{2}^{2}}{2 t} z}
$$

if $m=0$ and

$$
U(z)= \begin{cases}b-C_{+} e^{R_{+} z}-C_{-} e^{R_{-} z} & R_{+} \neq R_{-} \\ b-C_{0} e^{R_{z}}-C_{1} z e^{R_{z}} & R_{+}=R_{-}=R\end{cases}
$$

if $m \neq 0$. Here,

$$
R_{ \pm}=\frac{-\Gamma \pm \sqrt{\Gamma^{2}+m k_{2}^{2}}}{m} .
$$

If we impose the natural requirements that $U$ and $U^{\prime}$ are continuous and $U \geq 0$ and bounded, which apply if $U$ is the density of some quasiparticle, a probability, or a species population, and furthermore require that $U(0)=a$, we obtain explicit expressions from the above. We analyze them in the next two sections.

\section{DAMPED HARMONIC OSCILLATORS OF NEGATIVE MASS AND SPECIAL VALUES OF THE WAVEFRONT SPEED}

We introduce two new quantities $U_{R}$ and $U_{L}$ via

$$
U_{R}(z) \equiv U(z), \quad z \geq 0 ; \quad U_{L}(-z) \equiv b-U(z), \quad z \geq 0 .
$$

Equations (II.5) can then be written formally as the evolution equations describing two damped harmonic oscillators one of which has positive mass while the other has negative mass:

$$
\begin{aligned}
& m U_{R}^{\prime \prime}+2 \Gamma U_{R}^{\prime}+k_{1}^{2} U_{R}=0 \\
& -m U_{L}^{\prime \prime}+2 \Gamma U_{L}^{\prime}+k_{2}^{2} U_{L}=0
\end{aligned}
$$

The wavefront shape is given by $U_{R}(x-c t)$ for $x \geq c t$ and by $b-U_{L}(c t-x)$ for $x \leq c t$. Several interesting results emerge immediately.

Because $u$ describes a density or population, it cannot be negative. It follows that, if $m$ is positive, the damped harmonic oscillator representing the wavefront shape for $x \geq c t$ must be overdamped or critically damped. This 
is necessary because, the equilibrium value of that oscillator amplitude being zero, underdamping would make the amplitude go negative. The condition of no underdamping on the equation for $U_{R}$ in (III.2), viz., $\Gamma \geq k_{1} \sqrt{m}$, sets the requirement that the wavefront speed must exceed or equal a minimum value $c_{\min }$ given by

$$
c_{\min }=v\left[1+a(\alpha / 2 s)^{2}\right]^{-1} .
$$

Our result (III.3) is a generalization to finite correlation times of the minimum speed result known in the literature in the context of diffusive transport to which (III.3) reduces in the limit $\alpha \rightarrow \infty, s \rightarrow \infty, s^{2} / \alpha=k, v^{2} / \alpha=D$. This diffusive extreme of (III.3) is

$$
c_{\min }=2 \sqrt{k D / a}
$$

For this positive $m$ case we are first considering, in which the wavefront speed $c$ never exceeds the medium dictated speed $v$, the oscillator describing the left side of the wavefront shape has negative mass (see equation (III.2)). The slope assumed at $z=0$ is negative. The situation is thus that of a negative mass particle thrown towards its 'equilibrium' point, its momentum being depleted by the anti-restoring action of the force until the particle comes to rest at the 'equilibrium' point. If the particle were to overshoot the equilibrium point, the negativity of the mass would send it infinitely far from the equilibrium point. Since the corresponding infinite solutions are of no interest in our present investigation, we conclude that the behavior of $U_{L}$ shows no oscillations.

If we now consider the case when the wavefront speed exceeds the medium dictated speed, $m$ is negative, and the $U_{R}$ oscillator in (III.2) has negative mass. As in the $U_{L}$ case above, there are no oscillations on the right side of the wavefront shape. The $U_{L}$ oscillator now has positive mass. It can be underdamped since there is no problem in the density exhibiting oscillations around the positive value $b$. The condition of critical damping for this left oscillator is $\Gamma=\sqrt{|m|} k_{2}$, the corresponding wavefront speed being

$$
c_{o s c}=v\left[1-(b-a)(\alpha / 2 s)^{2}\right]^{-1} .
$$

If the speed exceeds $c_{o s c}$, the wavefront shape exhibits oscillations in space.

For any given set of the system parameters $v, \alpha, s, a, b$, there is always a minimum speed $c_{\min }$ given by (III.3). However, the existence of $c_{o s c}$, i.e., of shape oscillations, requires a condition to be satisfied by the parameters:

$$
2 s>\alpha(b-a)
$$

If (III.6) is not satisfied, $c_{o s c}$ is effectively infinite, and there are no oscillations in the wave shape. Also, in the limit $\alpha \rightarrow \infty, s \rightarrow \infty, s^{2} / \alpha=$ constant, $v^{2} / \alpha=$ constant, which corresponds to diffusive transport analyzed in the earlier literature, $c_{o s c}$ becomes infinitely large signifying that spatial oscillations never set in. For the opposite transport extreme in which $\alpha=0$, and the linear part of the evolution is an undamped wave equation, (III.3) and (III.5) show that $c_{o s c}, c_{\min }$ and the medium speed $v$ all become identical to one another. The shape always exhibits oscillations. Fig. 2 shows the dependence of $c_{\text {osc }}$ and $c_{\min }$ on the damping rate $\alpha / 2 s$. While our (III.3) is merely a generalization to finite correlation times of (III.4) known in the literature earlier, our results concerning the possible existence of $c_{o s c}$ and of spatial oscillations, in particular (III.5) and (III.6), are entirely new. They stem from the wave component of the telegrapher's equation manifested in the spatial oscillations of the wavefront shape.

Explicit solutions for the various cases in terms of the parameters in (II.6) are best presented by appealing to Fig. 3, which describes the positioning of the wavefront speed relative to the three characteristic values (in ascending order) $c_{\min }, v, c_{o s c}$. In region $\mathrm{I}, v>c>c_{\min }, m$ is positive, and we obtain from (II.8)

$$
U(z)=\left\{\begin{array}{ll}
A_{+} e^{r_{+} z}+A_{-} e^{r_{-} z} & z \geq 0 \\
b-(b-a) e^{R_{+} z} & z \leq 0
\end{array},\right.
$$

where

$$
\begin{aligned}
& A_{+}=\frac{a r_{-}+(b-a) R_{+}}{r_{-}-r_{+}}, \\
& A_{-}=\frac{a r_{+}+(b-a) R_{+}}{r_{+}-r_{-}} .
\end{aligned}
$$


In region $\mathrm{I}, c_{o s c}>c>v, m$ is negative, and

$$
U(z)=\left\{\begin{array}{ll}
a e^{r_{+} z} & z \geq 0 \\
b-C_{+} e^{R_{+} z}-C_{-} e^{R_{-} z} & z \leq 0
\end{array},\right.
$$

where

$$
\begin{aligned}
& C_{+}=\frac{(b-a) R_{-}+a r_{+}}{R_{-}-R_{+}}, \\
& C_{-}=\frac{(b-a) R_{+}+a r_{+}}{R_{+}-R_{-}} .
\end{aligned}
$$

In region III, $c>c_{\text {osc }}$ and $R_{ \pm}$is complex, giving rise to spatial oscillations on the left side. We express $R_{ \pm}$as $R \pm i 2 \pi / \lambda$ and obtain

$$
U(z)= \begin{cases}a e^{r+z} & z \geq 0 \\ b-\frac{b-a}{\cos \delta} e^{R z} \cos \left(\frac{2 \pi}{\lambda} z-\delta\right) & z \leq 0\end{cases}
$$

The wavelength $\lambda$ and the phase $\delta$ are given by

$$
\begin{gathered}
\lambda=\frac{4 \pi\left(c^{2}-v^{2}\right)}{\sqrt{4\left(c^{2}-v^{2} k_{2}^{2}-\alpha^{2} c^{2}\right.}}, \\
\delta=-\tan ^{-1}\left(\frac{\lambda}{2 \pi(b-a)}\left[a r_{+}+(b-a) R\right]\right) .
\end{gathered}
$$

At the three boundaries of the regions,

$$
U(z)= \begin{cases}a e^{r z}+\left(a r-(b-a) R_{+}\right) z e^{r z} & z \geq 0 \\ b-(b-a) e^{R_{+} z} & z \leq 0\end{cases}
$$

when $c=c_{\min }$;

$$
U(z)= \begin{cases}a e^{-\left(k_{1}^{2} / 2 \Gamma\right) z} & z \geq 0 \\ b-(b-a) e^{\left(k_{2}^{2} / 2 \Gamma\right) z} & z \leq 0\end{cases}
$$

when $c=v$; and

$$
U(z)= \begin{cases}a e^{r+z} & z \geq 0 \\ b-(b-a) e^{R z}+\left(a r_{+}+(b-a) R\right) z e^{R z} & z \leq 0\end{cases}
$$

when $c=c_{\text {osc }}$.

The above solutions, whose two typical shapes (with and without spatial oscillations) are shown in Fig. 4, allow us to examine analytically the relationship between the shape of the wavefront and its dynamics, specifically the speed dependence of the front steepness and of the front wavelength. Since the slopes are matched at $z=0$, we may use either the $z \geq 0$ or $z \leq 0$ expressions from the explicit solutions according to convenience. We see that the slope is given by $a r_{+}$when $c>v$ and by $-(b-a) R_{+}$when $c<v$. Using the definitions for $r_{+}$in (II.8) and for $R_{+}$in (II.11), we have

$$
U^{\prime}(0)= \begin{cases}-(b-a)\left[-\Gamma+\sqrt{\Gamma^{2}+m k_{2}^{2}}\right] / m & c<v \\ -a k_{1}^{2} / 2 \Gamma & c=v . \\ a\left[-\Gamma+\sqrt{\Gamma^{2}-m k_{1}^{2}}\right] / m & c>v\end{cases}
$$


The slope-speed relation (III.15) that we obtain in the presence of finite transport correlation times is found to have a considerably richer structure than what is known in the diffusive extreme. In that extreme, the slope is a monotonically decreasing function of the speed:

$$
U^{\prime}(0)=-(b-a) \frac{c}{2 D}\left(-1+\sqrt{\frac{4 D k}{(b-a) c^{2}}}\right)
$$

This simple behavior is seen in the inset in Fig. 5. For finite $\alpha$, however, the slope can display nonmonotonic behavior as seen in the main Fig. 5. There is a kink in the slope-speed plot at $c=v$. For the region in which the wavefront speed is larger than the medium-dictated speed, the slope decreases with increasing speed: At the point the two speeds equal each other, there is a discontinuity in the curvature. If the wavefront speed is smaller than $v$, we find that two subregions have to be considered according as to whether the inequality

$$
\alpha^{2}(b-a) \geq 2 s^{2}
$$

is, or is not, satisfied. If it is satisfied, the slope continues to increase. Otherwise, the graph appears similar to a parabola whose minimum occurs when

$$
c=c_{*} \equiv \frac{\sqrt{b-a}}{2 s} \alpha c_{o s c}
$$

Fig. 6 clarifies the various shapes the slope-speed relation can exhibit in different ranges of the parameter $c_{*}$. In the diffusive limit, the location of the minimum tends to infinity on the $c$-axis, with the result that two of the three regions disappear: $c$ is always smaller than $v$ (which tends to infinity in the diffusive limit), and a monotonic decrease of the slope is all that remains. The introduction of finite correlation times into the reaction diffusion problem, which is the purpose of the present study, is thus seen to uncover an unexpectedly large variety of behavior in the shape-speed dependence of the wavefront. We present Fig. 7 to show how this correlation time (equivalently its reciprocal, the damping parameter $\alpha$ ) controls the slope.

In the case when spatial oscillations are present in the wavefront, the dependence of the oscillation wavelength on the wave speed is given by (III.10). We plot it in Fig 8 . We see that the behavior is not monotonic there being a minimum value of the wavelength.

The solutions given above deal exclusively with fronts that decay to zero at their leading edge. For the most part, these are the only fronts that exist, because solutions that do not decay to zero as $z \rightarrow \infty$ tend to diverge. However, if we have an oscillating solution, the divergence need not necessarily occur. We can then have nondecaying wavetrains that extend to infinity in each direction. There is no unique solution associated with each speed, each solution has a different slope at $z=0$, and the previous discussions on the relation between steepness and speed have no relevance to these wavetrain class of solutions. Nevertheless, the speed does determine the wavelengths $\lambda$. If the correlation time is infinite, i.e., in the limit $\alpha \rightarrow 0$, we have $c_{\min }=c_{o s c}=v$, and the solution becomes a step-function $U(z)=b \Theta(-z)$ when $c=v$. When $c>v$, the solution is either a pulse or a wavetrain. The pulse solution is symmetric about its maximum and may be determined analytically:

$$
U(z)= \begin{cases}a e^{-\mu_{+} z} & z \geq 0 \\ b-\frac{b-a}{\cos \delta} \cos \left(\frac{2 \pi}{\lambda} z-\delta\right) & z_{1} \leq z \leq 0 \\ a e^{\mu\left(z-z_{1}\right)} & z \leq z_{1}\end{cases}
$$

where

$$
\delta=-\tan ^{-1}\left(\sqrt{\frac{a}{b-a}}\right), z_{1}=2 \delta,
$$

and the wavelength of the oscillating term is given by

$$
\lambda=\frac{1}{2 \pi k_{2}} \sqrt{c^{2}-v^{2}}
$$

In contrast to (III.10) and Fig 8, we see that, in the case of these infinite wavetrains, the wavelength does increase monotonically with speed.

The pulse solution and a wavetrain solution, which is determined numerically, are displayed in Fig. 9. The two have identical wavelengths in the oscillating regime. 


\section{DISCUSSION}

In this concluding section we discuss the original motivation for the research reported in this paper, and summarize our primary results.

Transport equations such as the telegrapher's equation with nonlinear additive terms as in (II.2) have appeared recently [4] in our analysis of the distribution of stress in granular compacts. The memory, which is spatial in the granular context rather than temporal as in the present analysis, arises from characteristics of the granular material such as grain size, grain shape and friction. The nonlinear terms arise from the feedback provided by the dependence of the material density on the stress [5]. Our original motivation for this work stemmed from the possibility that the combined effect of the memory and nonlinearity on the stress distribution might be related to arches in granular compacts. The coordinate $t$ in the present analysis corresponds to the spatial coordinate in the vertical direction (the direction of gravity and/or that of the applied pressure) in the granular compact. The quantity $u$ here refers to the stress in the compact. The specific form of the nonlinear terms derived in [4] is not that of $f(u)$ and does not appear to lead to the formation of nonlinear waves. However, it is known [9] that granular material columns which are tall enough are selfsupporting as a result of the friction along the sides of the container. This observation leads on qualitative grounds to equations of the type analysed in the present paper. Nevertheless, because important issues concerning the relevance of the analysis to actual observations in granular materials remain to be clarified, we postpone a detailed discussion in the granular context.

The point of departure of the present paper is (II.2). The primary results we have obtained are of two kinds. The first group constitutes generalizations of results known earlier for the diffusive extreme but now extended to cover finite correlation times. The second group contains results not known earlier. To the first group belong the appearance of nonlinear wavefronts, the generalized form of the minimum speed that the wavefronts take, particularly its dependence (III.3) on the nonlinearity parameters $a$ and $s$ as well as on the correlation parameter $\alpha$, and the unexpectedly rich relationship (III.15) of the speed of the wavefront to its shape with explicit dependence on the correlation time. To the second group belong the possible existence of a speed beyond which spatial oscillations appear in the wavefront shape, the corresponding expression (III.5) and the condition (III.6), the relationship (III.10) of the speed of the wavefront to the wavelength of the spatial oscillations, and the appearance and characteristics of wavetrains of infinite extension. Other interesting features of the analysis include the emergence of the damped harmonic oscillators with negative mass which facilitate an understanding of the results. Basic to the fact that our analysis is analytical is the approach [10] of the piece-wise linear representation of the nonlinearity. We mention in passing that, so long as the memory function used in the analysis is Markoffian, i.e., has a finite value for its integral $\int_{0}^{\infty} \phi(t)$, there are no additional contributions to pattern formation of our analysis over earlier results [10] based on the diffusive extreme. The generalization of the usual reaction-diffusion equation (1.4) to the form (II.2) that we have used in the present investigation, is obviously not unique, our present choice having been dictated by analytic tractability.

In closing, we raise the following question. Is it perhaps possible that, when observations in systems are interpreted on the basis of linear intuition to correspond to certain signal speeds, and conclusions are drawn on the basis of the observations about the medium speed $v$, gross overestimates or underestimates might be occuring because the system is nonlinear, the signal speed being $c$ rather than $v$ ? It is to be noted that the nonlinear speed $c$ can have an arbitrarily large value dictated only by the wavefront shape.

This work was supported in part by Sandia National Laboratories under Department of Energy Contract DE-AC04$94 \mathrm{~A} 85000$.

[1] R. A. Fisher, Ann. Eugenics 7, 353 (1937).

[2] J. D. Murray, Mathematical Biology, 2nd edition (Springer, New York, 1993).

[3] V. M. Kenkre and P. Reineker, Exciton Dynamics in Molecular Crystals and Aggregates, (Springer, Berlin, 1982); V. M. Kenkre in Proceedings of the NATO Advance Study Institute on Energy Transfer, Erice, Italy, June 15-30, 1983, ed. B. DiBartolo, (Plenum, New York, 1984).

[4] V. M. Kenkre, J. Scott, E. Pease and A. J. Hurd, Phys. Rev. E 57, 5841 (1998); J. Scott, V. M. Kenkre and A. J. Hurd, Phys. Rev. E 57, 5850 (1998).

[5] I. Aydin, B. J. Briscoe, and K. Y. Sanliturk, Computational Materials Science 3, 55 (1994); Powder Technology 89, 239 (1996); H. M. Macleod and K. Marshall, Powder Technology 16, 107 (1977).

[6] P. Duwez and L. Zwell, Trans. AIME 185, 137 (1949); D. Train, Trans. Instn. Chem. Engrs. 35, 258 (1957).

[7] We are not referring here to spatial regions but to ranges of the magnitude of the wave amplitude $U$. 
[8] V. M. Kenkre, M. R. Endicott, S. J. Glass and A. J. Hurd, J. Am. Ceramic Soc. 79, 3045 (1996).

[9] H. A. Janssen, Z. Ver. Dt. Ing. 39, 1045 (1895); R. A. Thompson, Ceramic Bulletin 60, 237, 244, 248 (1981).

[10] D. E. Strier, D. H. Zanette, and H. S. Wio, Physica A 226, 310 (1995); G. Izus, R. Deza, C. Borzi, and H. S. Wio, Physica A 237, 135 (1997).

FIG. 1. The nonlinearity function $f(u)$ in the piece-wise linear form used for analytic calculations in the present paper (solid line). The Fisher term (dashed line) is symmetrical but in our analysis we take $a$, the value of $u$ at which the nonlinerity peaks, and $b$, the second zero of $f(u)$, to be independent of each other.

FIG. 2. The dependence of the respective ratios of $c_{\min }$ and $c_{\text {osc }}$ to the medium dictated speed $v$ on the damping-nonlinearity parameter ratio $\alpha / 2 s$. It is seen that the minimum speed decreases monotonically. The oscillatory speed has, however, a characteristic value of $\alpha / 2 s$ at which it blows up. Beyond that value, the oscillatory speed is, in effect, infinite.

FIG. 3. Regions of the wavefront speed c. The speed cannot be lower than $c_{\min }$. Solutions have different shapes according to whether $c$ lies between $c_{\min }$ and the medium dictated speed $v$, between the latter and $c_{o s c}$, larger than $c_{o s c}$, or on the boundaries separating these regions. The explicit expressions are displayed in the text in reference to these regions.

FIG. 4. Two examples of the wavefront shape: (a) without and (b) with spatial oscillations. Values of the parameters are arbitrary: $v=10, \alpha=5, s=3, a=1, b=2$, in both (a) and (b); the speed $c=9$ in (a) and $c=18$ in (b).

FIG. 5. The variety of behavior of the slope-speed relationship in the presence of finite transport correlation times. The inset (with coordinates on both axes identical to those on the main figure) shows the diffusive limit which is well known in the literature.

FIG. 6. Different dependences of the slope on the speed according to the value of the parameter $c_{*}$. See text for details.

FIG. 7. The dependence of the slope of the wavefront on the transport correlation time. Plotted is $\left|U^{\prime}(0)\right|$ versus $\alpha$ for $v=10, s=3, a=1$, and $b=2$.

FIG. 8. Dependence of the wavelength $\lambda$ on the wavefront speed for pulse solutions for the case in which spatial oscillations occur.

FIG. 9. Wavetrain solutions extending to infinity in each direction found by numerically determing the coefficients of the general solution. The parameters are $v=10, \alpha=0, s=3, a=1$, and $b=2$. The inset (with coordinates on both axes identical to those on the main figure) depicts the wavepulse solution for the same parameters. 


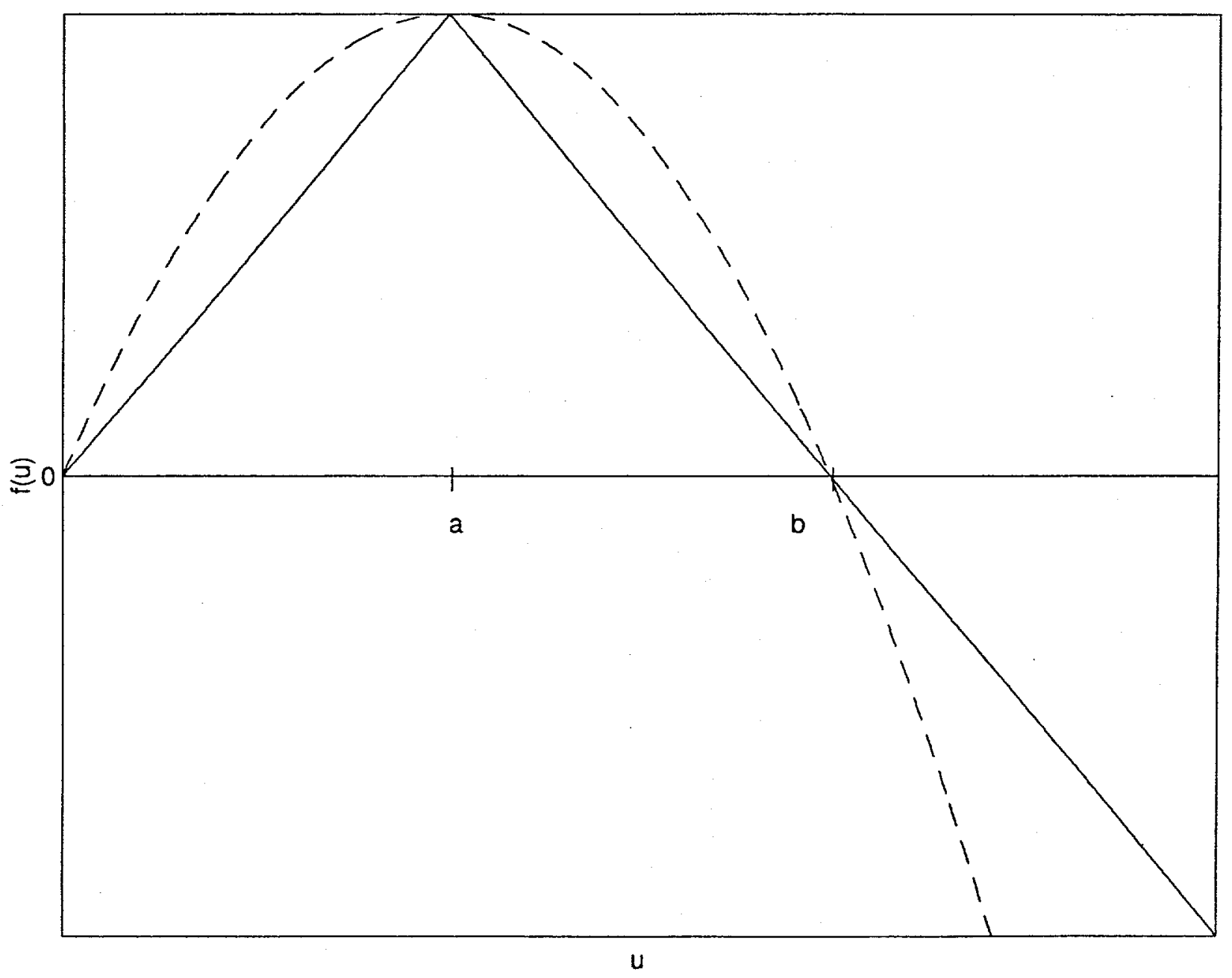




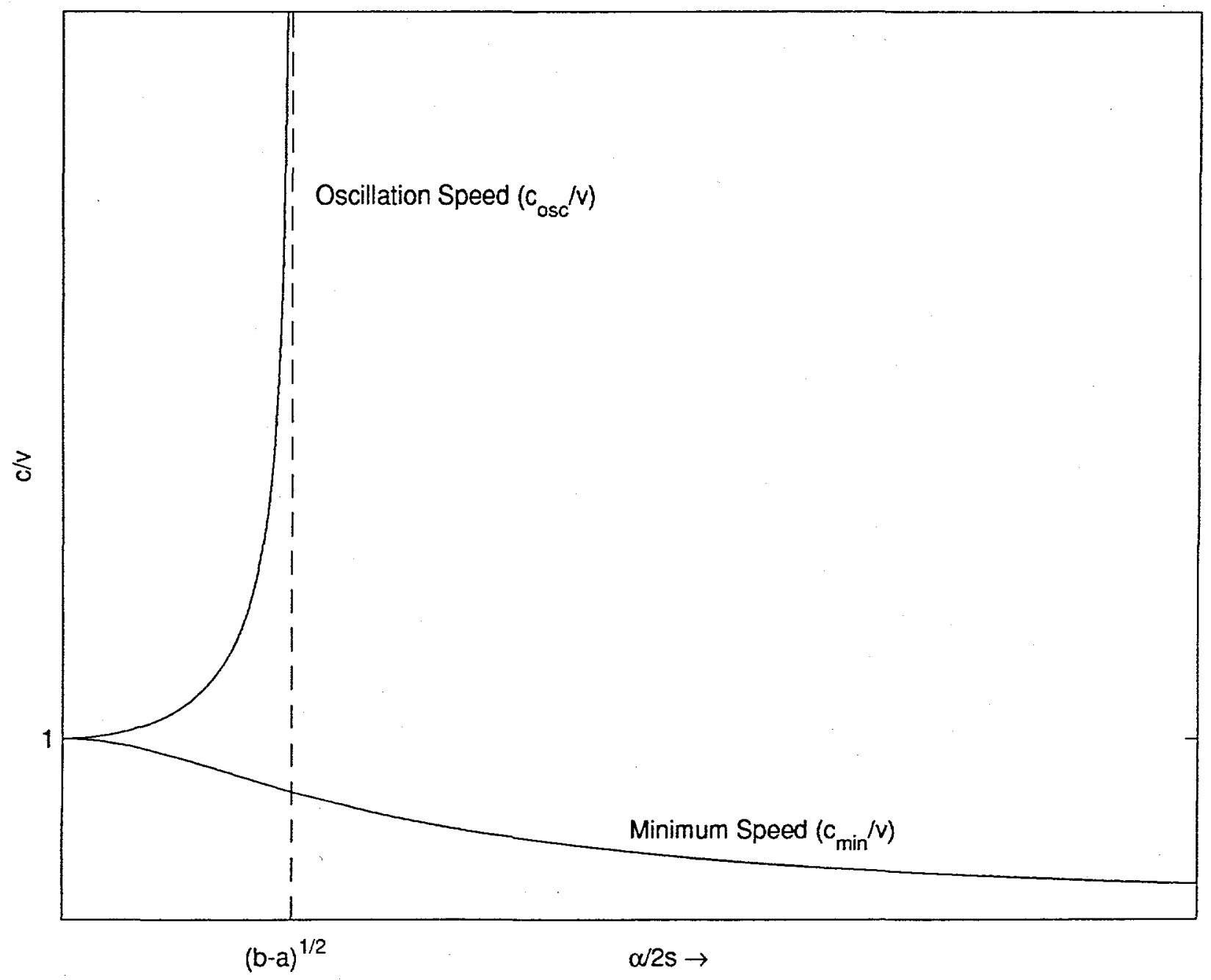




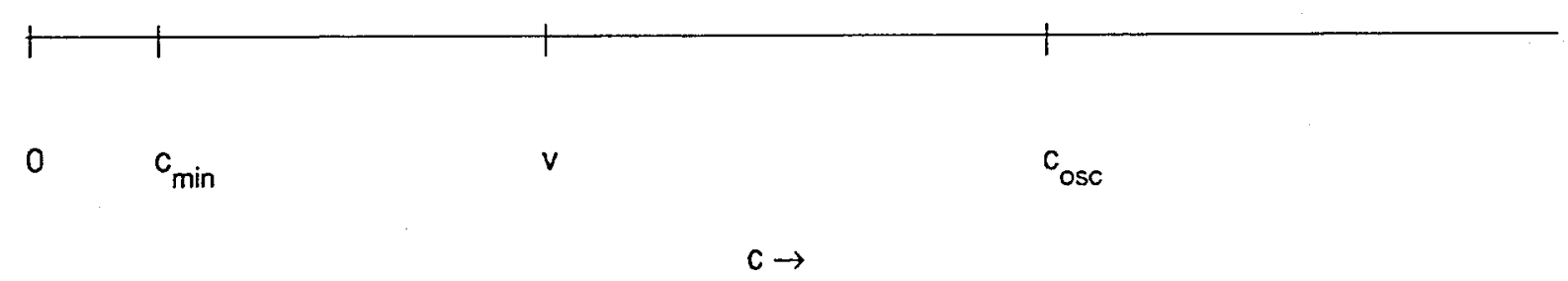



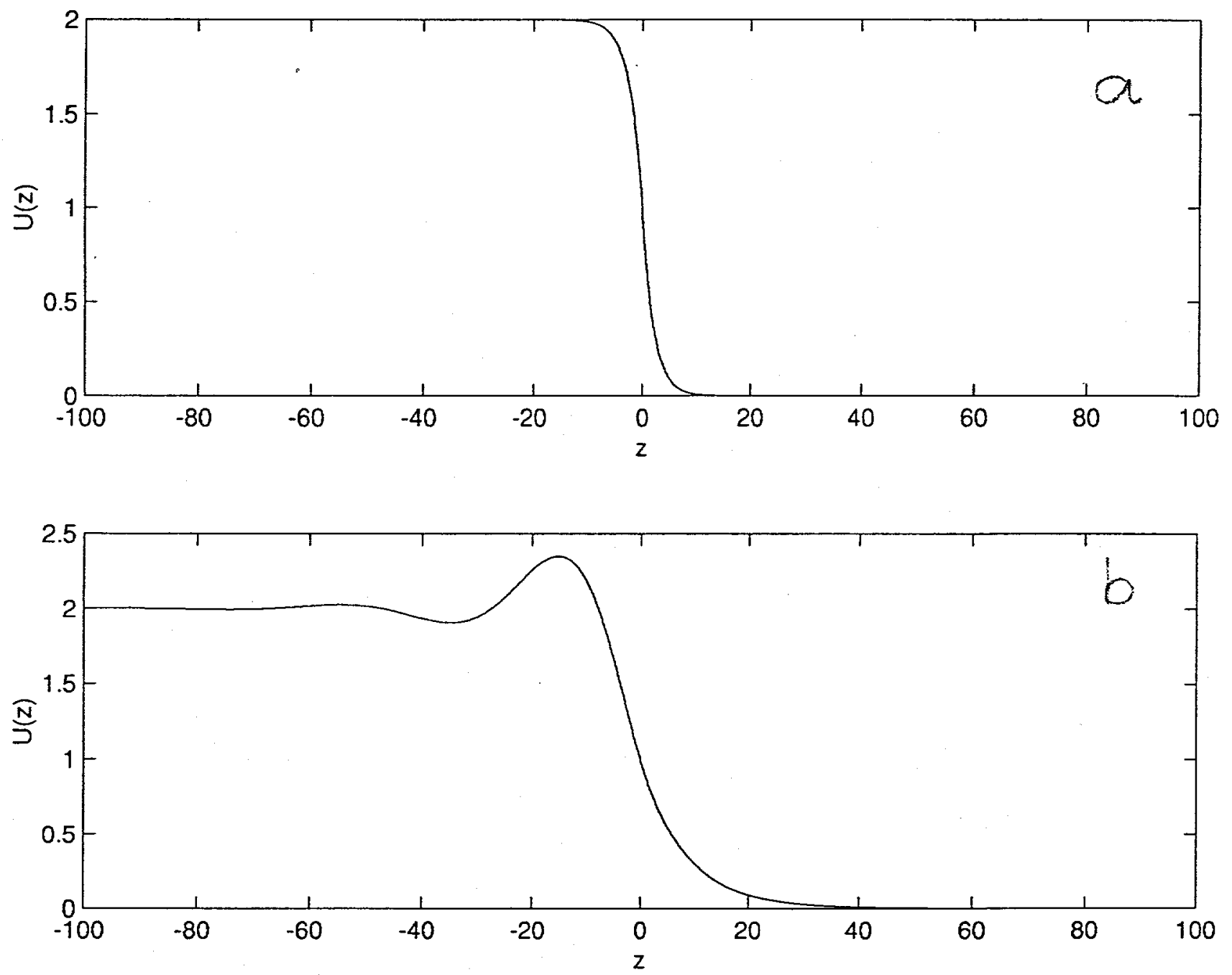


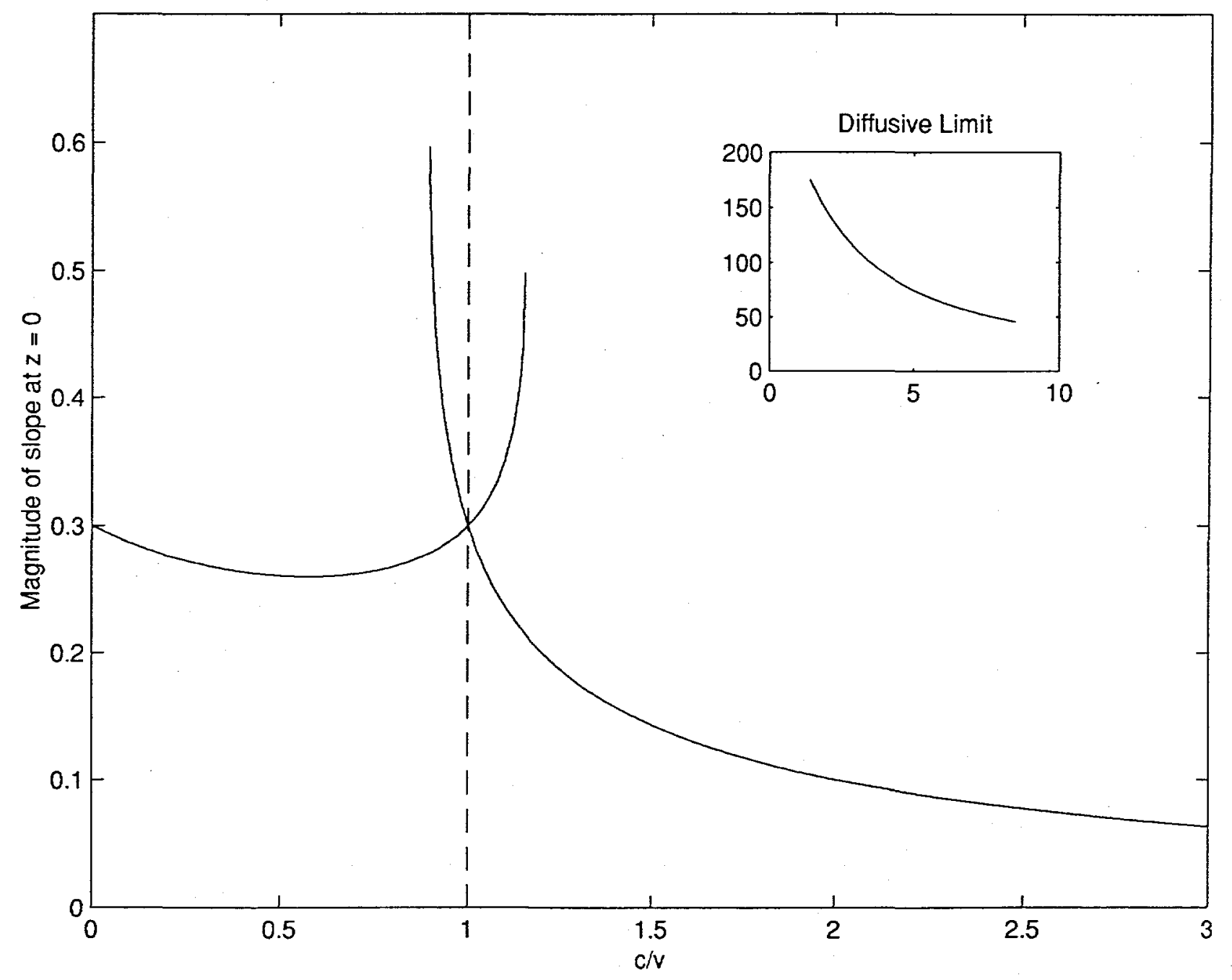




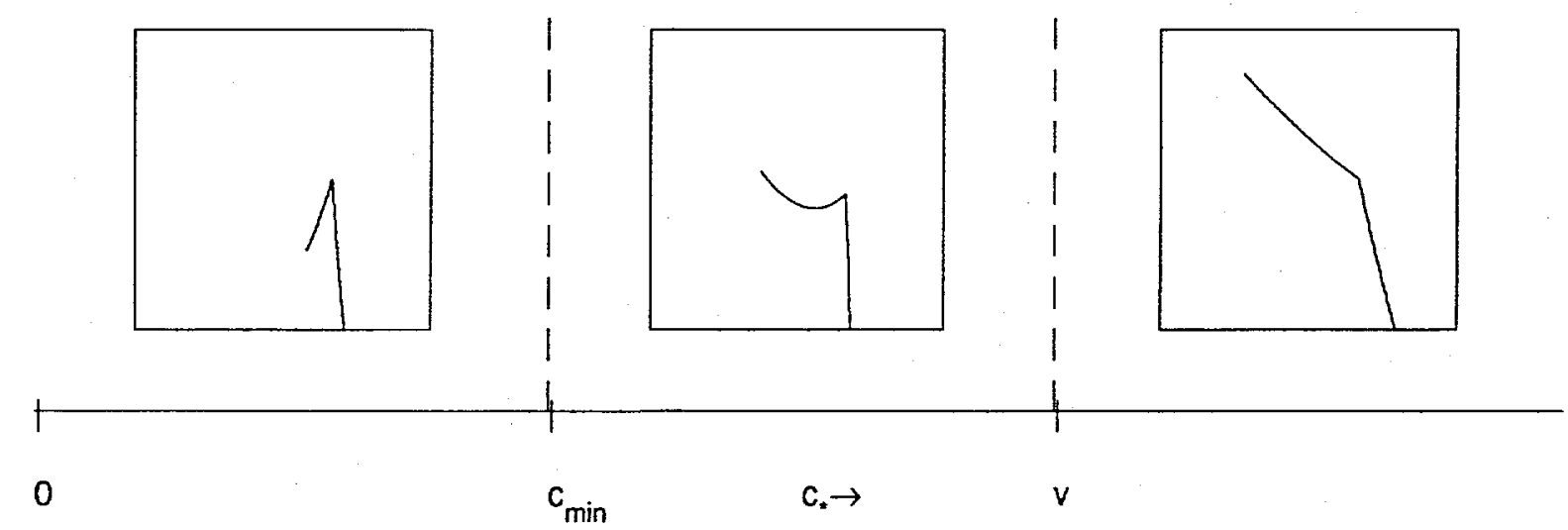




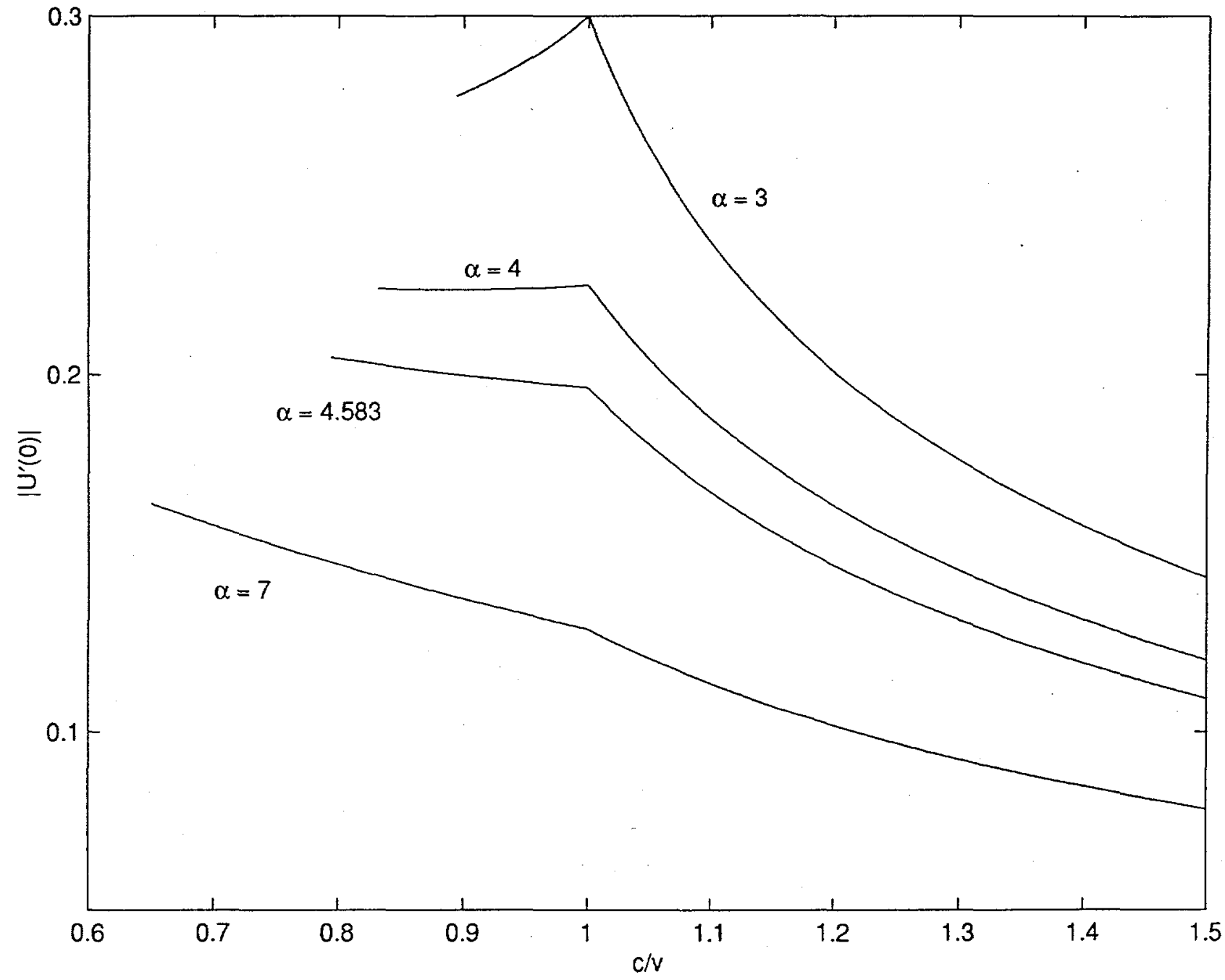




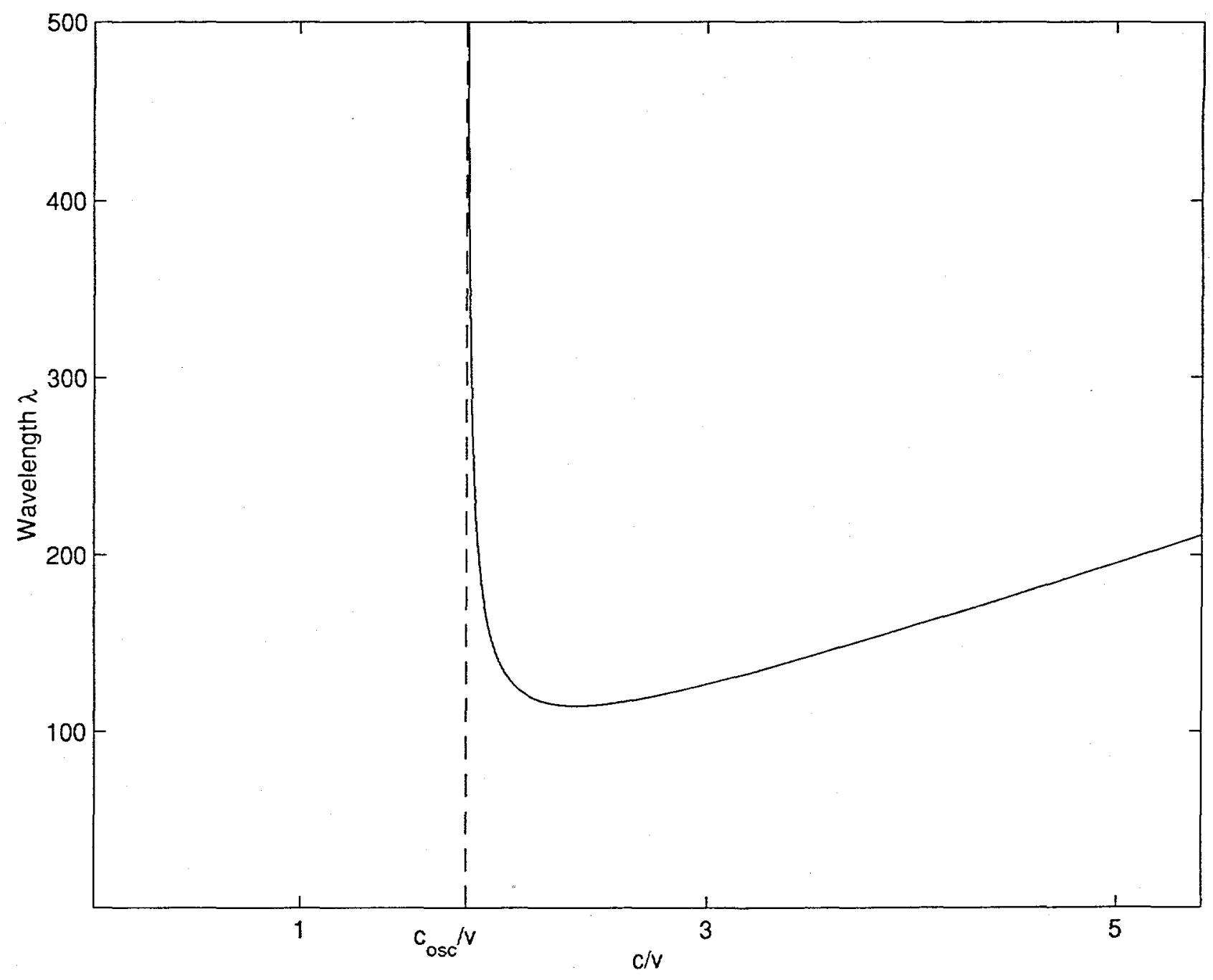




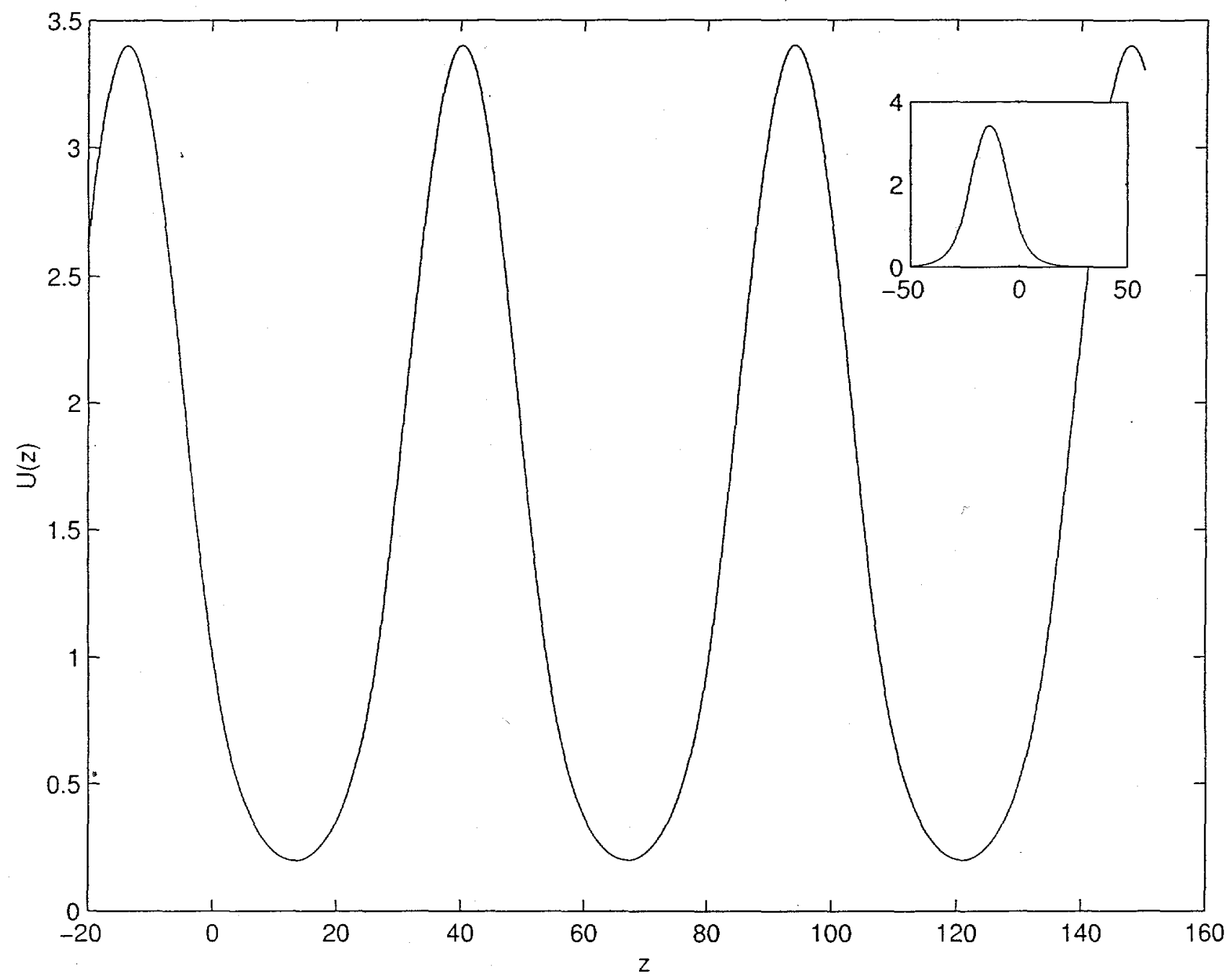

